\title{
APPROXIMATION BY SMOOTH MULTIVARIATE SPLINES
}

\author{
BY \\ C. DE BOOR ${ }^{1}$ AND R. DEVORE ${ }^{2}$
}

\begin{abstract}
The degree of approximation achievable by piecewise polynomial functions of given total order on certain regular grids in the plane is shown to be adversely affected by smoothness requirements - in stark contrast to the univariate situation. For a rectangular grid, and for the triangular grid derived from it by adding all northeast diagonals, the maximum degree of approximation (as the grid size $1 / n$ goes to zero) to a suitably smooth function is shown to be $O\left(n^{-\rho-2}\right)$ in case we insist that the approximating functions are in $C^{\rho}$. This only holds as long as $\rho \leqslant(r-3) / 2$ and $\rho \leqslant(2 r-4) / 3$, respectively, with $r$ the total order of the polynomial pieces. In the contrary case, some smooth functions are not approximable at all. In the discussion of the second mesh, a new and promising kind of multivariate B-spline is introduced.
\end{abstract}

1. Introduction. One of the important properties of univariate splines is that in most senses smooth splines approximate just as well as piecewise polynomials. As we shall see, this is no longer the case for multivariate splines where both the smoothness of the spline and the geometry of the partition can have a limiting effect on the order of approximation. This type of limitation has already been recognized in certain cases [9], such as low total order splines on a rectangular grid. For example, there is no effective approximation by $C^{(1)}$-cubics (i.e., total order 4 ) on rectangular grids. The purpose of this paper is to give a systematic study of this and related questions. We restrict our inquiry to bivariate approximation but it will be clear that our techniques extend to higher dimensions.

We became involved in these questions because we wanted to compare the relative merits of coordinate order splines with those of total order splines. It is well known that $C^{(r-2)}$-smooth splines of coordinate order $r$ on a rectangular grid of mesh size $h$ approximate smooth functions to within $O\left(h^{r}\right)$. The same order is achieved by piecewise polynomials of coordinate order $r$ on the same grid. On the other hand, piecewise polynomials of total order $r$ on the same grid also approximate smooth functions to within $O\left(h^{r}\right)$, but with a considerable savings in the number of local degrees of freedom used: $r(r+1) / 2$ versus $r^{2}$. It is natural to ask whether this state of affairs persists when we impose some smoothness on the approximating functions. The answer is a resounding "no"; any smoothness requirement whatever will

Received by the editors December 21, 1981 and, in revised form, April 7, 1982.

1980 Mathematics Subject Classification. Primary 41A15, 41A63, 41A25.

Key words and phrases. Multivariate, splines, piecewise polynomial, smoothness, degree of approximation, B-splines.

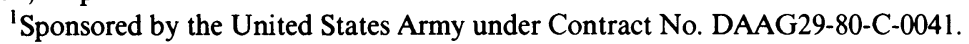

${ }^{2}$ Supported by NSF Grant No. MCS 81-01661. 
adversely affect the rate of approximation by total order splines on a rectangular grid. This is true for other partitions as well. This connection between smoothness requirements, the geometry of the partition and the order of approximation achievable is the main theme of this paper.

Let $\Pi=\left\{\pi_{i}\right\}$ be a partition of $\mathbf{R}^{2}$ into triangles and/or rectangles. Denote by $\Pi_{n}:=\left\{\pi_{i}(n)\right\}$ the corresponding scaled partition, with $\pi_{i}(n):=\pi_{i} / n$, all $i$. Denote by $S_{r, \rho}\left(\Pi_{n}\right)$ the space of splines of order $r$ and smoothness $\rho$ on $\Pi_{n}$, i.e., $s \in S_{r, \rho}\left(\Pi_{n}\right)$ if and only if $s \in C^{\rho}\left(\mathbf{R}^{2}\right)$ and, on each $\pi_{i}(n), s$ is a polynomial of total order $r$ (i.e. total degree $<r)$. We are interested in when $\cup_{n=1}^{\infty} S_{r, \rho}\left(\Pi_{n}\right)$ is dense in $C_{0}\left(\mathbf{R}^{2}\right)$. We study this problem in detail for two particular partitions: $\Sigma:=\left\{\sigma_{i j}\right\}$, consisting of the squares $\sigma_{i j}:=[i, i+1] \times[j, j+1]$, and the partition $\Delta$ which results when each square of $\Sigma$ is divided into two triangles by introducing the northeast diagonal. The technique developed for these two cases can be used for more general partitions as well.

In $\S 2$, we study approximation on $\Sigma_{n}$. This modest example already has the salient features of the general problem. We show that $S_{r, \rho}\left(\Sigma_{n}\right)$ is effective if and only if $\rho \leqslant(r-3) / 2$. Thus, roughly speaking, only smoothness up to one-half the order of the polynomial pieces is allowable in this case. It turns out that smoothness also affects the rate of approximation in that certain $C_{0}^{\infty}$-functions can only be approximated to within $O\left(n^{-r+\rho+1}\right)$. Thus any smoothness condition reduces the achievable order of approximation. This should be compared with the univariate case or the tensor product case where the full order of approximation is achievable regardless of smoothness.

We study approximation on $\Delta_{n}$ in $\S 3$. Here, the role of the geometry of the partition becomes more apparent. The splines $S_{r, \rho}\left(\Delta_{n}\right)$ are effective if and only if $\rho \leqslant(2 r-4) / 3$, hence there is a gain over the case $\Sigma_{n}$ from roughly $r / 2$ to $2 r / 3$. It is clear from our techniques that this is due to the fact that the partition $\Delta$ has three pairwise independent directions, viz. $(1,0),(0,1)$ and $(1,1)$, whereas $\Sigma$ has only two, viz. $(1,0)$ and $(0,1)$. More generally, if the partition $\Pi$ is generated by $m$ pairwise independent vectors (what we mean by this is made precise in $\S 4$ ), then $S_{r, \rho}\left(\Pi_{n}\right)$ is effective if and only if $\rho \leqslant r-1-\lceil(r+1) / m]$. Thus the more directions, the higher the allowable smoothness, but of course at the expense of a more complicated partition.

The results just described have two components. First they say that $\cup_{n} S_{r, \rho}\left(\Pi_{n}\right)$ is not dense in $C_{0}\left(\mathbf{R}^{2}\right)$ if $\rho$ is too large. This rests on the fact that $S_{r, \rho}\left(\Pi_{n}\right)$ will not contain splines of finite support when $\rho$ is too large. Our approach for this part of the problem is more or less the same in both cases $\Pi=\Sigma$ and $\Pi=\Delta$. The second half of the analysis is to show that $\cup_{n} S_{r, \rho}\left(\Pi_{n}\right)$ is dense when $\rho$ is suitably restricted. This requires the construction of appropriate approximation methods. We develop different methods for constructing such approximations in the two cases.

For $\Sigma$ it is easy to see that $S_{n}:=S_{r, \rho}\left(\Sigma_{n}\right)$ is effective when $\rho \leqslant(r-3) / 2$ since $S_{n}$ then contains the tensor products of univariate splines of order $\rho+2$ and smoothness $\rho$. The more difficult problem is to show that $S$ approximates any $C_{0}^{\infty}$-function $f$ to within $O\left(n^{-r+\rho+1}\right)$. This is done by approximating the derivative $D^{(1,1)} f$ by splines 
of lower order and smoothness, integrating and making local corrections for the approximation to $f$.

Our construction of approximants from $S:=S_{r, \rho}\left(\Delta_{n}\right)$ has a completely different flavor, being based on extensions of the idea of multivariate B-splines. These ideas extend readily to more general partitions. Recall that multivariate B-splines are defined by certain cross sectional volumes of simplices. If the simplex is replaced by a more general polyhedron, the resulting function is still a piecewise polynomial, but now perhaps with fewer lines of discontinuity. In particular, by a suitable choice of the polyhedron, we can force the discontinuities of the resulting spline to be contained in the mesh lines of $\Delta_{n}$. As mentioned in $\$ 4$, other choices for the polyhedra will handle other partitions $\Pi$ of $\mathbf{R}^{2}$. We feel that this viewpoint for constructing multivariate splines may prove to be very useful. For example, some of the standard finite elements can be described this way [3].

Here are some notational conventions used. $C_{0}^{r}(A)$ stands for the set of $r$ times continuously differentiable functions on the set $A$ with compact support in $A$. $\|\cdot\|$ is the $L_{\infty}$-norm on $\mathbf{R}^{2}$, and $\|\cdot\|(A)$ is the $L_{\infty}$-norm over the set $A$. Further,

$$
\|f\|_{r}:=\|f\|_{W_{\infty}^{r}}:=\max _{\alpha+\beta \leqslant r}\left\|D^{(\alpha, \beta)} f\right\| \text { and }|f|_{r}:=\max _{\alpha+\beta=r}\left\|D^{(\alpha, \beta)} f\right\| .
$$

$\lfloor a\rfloor$ denotes the largest integer no bigger than $a$, i.e., the "floor" function, and $\lceil a\rceil$ denotes its companion, the "ceiling" function, which gives the smallest integer no smaller than $a$. Further,

$$
\mathbf{m}:=\{1,2, \ldots, m\} .
$$

We also need the difference operator $\Delta_{h}^{r}$ defined on the function $g$ by the rule

$$
\Delta_{h}^{r} g:(x, y) \mapsto \sum_{p, q=0}^{r}(-1)^{p+q}\left(\begin{array}{l}
r \\
p
\end{array}\right)\left(\begin{array}{l}
r \\
q
\end{array}\right) g(x+p h, y+q h) .
$$

This is the tensor product of the univariate $r$ th order difference operators in the coordinate directions. Explicitly, $\Delta_{h}^{r}=\delta_{(h, 0)}^{r} \cdot \delta_{(0, h)}^{r}$, with

$$
\delta_{(a, b)}^{r} g:(x, y) \mapsto \sum_{p=0}^{r}(-1)^{r-p}\left(\begin{array}{l}
r \\
p
\end{array}\right) g(x+p a, y+p b) .
$$

2. Approximation on rectangular grids. Let $\Sigma:=\left\{\sigma_{i j}\right\}$ with $\sigma_{i j}:=\left[x_{i}, x_{i+1}\right] \times$ $\left[y_{j}, y_{j+1}\right]$ and $x_{i}:=y_{i}:=i$. We are interested in the restriction of $S:=S_{r, \rho}(\Sigma)$ to some compact set.

LEMMA 1. Every $s \in S=S_{r, \rho}(\Sigma)$ can be represented on

$$
\mathbf{R}_{+}{ }^{2}:=\{(x, y): x, y \geqslant 0\}
$$

as a linear combination of the truncated powers in

$$
T_{r, \rho}(\Sigma):=\left\{\left(x-x_{i}\right)_{+}^{p}\left(y-y_{j}\right)_{+}^{q}: i, j, p, q \geqslant 0 ; p+q<r ;{ }_{q}^{p}>\rho \text { if }_{j}^{i}>0\right\} .
$$

Proof. This is proved by induction, similar to the proof that truncated power functions are a basis for univariate splines. 
Recall that $\Sigma_{n}$ denotes the partition $\Sigma$ scaled by $1 / n$. Let $x_{i}(n):=y_{i}(n):=i / n$. Set $S_{n}:=S_{r, \rho}\left(\Sigma_{n}\right)$.

THEOREM 1. $\cup_{n=1}^{\infty} S_{n}$ is dense in $C_{0}\left(\mathbf{R}^{2}\right)$ if and only if $\rho \leqslant(r-3) / 2$.

Proof. If $\rho \leqslant(r-3) / 2$, then $S_{n}$ contains all the truncated powers $(x, y) \mapsto$ $\left(x-x_{i}(n)\right)_{+}{ }^{\rho+1}\left(y-y_{j}(n)\right)_{+}{ }^{\rho+1}$ and hence the space $\tilde{S}_{\rho+2, \rho}\left(\Sigma_{n}\right)$ of splines of coordinate order $\rho+2$ and smoothness $\rho$. It is known that $\cup_{n} \tilde{S}_{\rho+2, \rho}\left(\Sigma_{n}\right)$ is dense in $C_{0}\left(\mathbf{R}^{2}\right)$; see for example [5].

Suppose that $\rho>(r-3) / 2$ and that $f \in C_{0}^{\infty}\left(\mathbf{R}^{2}\right)$ with $\left\|f-s_{n}\right\|=o(1)$ as $n \rightarrow \infty$, for some splines $s_{n} \in S_{n}$. Suppose without loss of generality that $f$ has its support in $\mathbf{R}_{+}{ }^{2}$. Since $\rho>(r-3) / 2$, the only truncated power functions in $T_{r, \rho}(\Sigma)$ are of the form $t(x, y)=x^{p}\left(y-y_{j}\right)_{+}^{q}$ or $t(x, y)=\left(x-x_{i}\right)_{+}{ }^{p} y^{q}$ with $p+q<r$. Since $\Delta_{h}^{r} t=$ 0 for such $t$, we have from Lemma 1 that $\Delta_{h}^{r} s=0$ on $\mathbf{R}_{+}^{2}$ for all $s \in S$. This implies that we also have $\Delta_{h}^{r} s_{n}=0$ on $\mathbf{R}_{+}{ }^{2}$ for $n=1,2, \ldots$, from which it follows that $\Delta_{h}^{r} f=0$. If we divide by $h^{2 r}$ and take the limit as $h \rightarrow 0$, we find that $D^{(r, r)} f=0$. Since there are $C_{0}^{\infty}$-functions for which $D^{(r, r)} f \neq 0$, we have proved the theorem.

Theorem 1 shows that only splines with smoothness less than about one-half the order $r$ will be effective for approximation. It turns out that even when $\rho \leqslant(r-3) / 2$, the order of approximation is affected negatively by smoothness. More precisely, we now show that the optimal order of approximation achievable with splines of order $r$ and smoothness $\rho$ is $n^{-r+\rho+1}$.

THEOREM 2. Let $\rho \leqslant(r-3) / 2$ and $k:=r-\rho-1$. Then

(i) there are functions $f \in C_{0}^{\infty}\left(\mathbf{R}^{2}\right)$ for which $\operatorname{dist}\left(f, S_{n}\right) \neq o\left(n^{-k}\right), n \rightarrow \infty$;

(ii) for each $f \in C_{0}^{\infty}\left(\mathbf{R}^{2}\right)$, $\operatorname{dist}\left(f, S_{n}\right)=O\left(n^{-k}\right)$.

Proof. We will show that if $f \in C_{0}^{\infty}\left(\mathbf{R}^{2}\right)$ and $\operatorname{dist}\left(f, S_{n}\right)=o\left(n^{-k}\right)$, then $D^{(r+k, r)} f$ vanishes at 0 , and this shows (i). Actually, with a finer analysis, we could show that all $k$ th order derivatives of $D^{(r, r)} f$ vanish on all of $\mathbf{R}^{2}$.

Suppose that $f \in C_{0}^{\infty}\left(\mathbf{R}^{2}\right)$ and that there are functions $s_{n} \in S_{n}$ such that

$$
\left\|f-s_{n}\right\|=o\left(n^{-k}\right) \text { as } n \rightarrow \infty .
$$

Let $m$ be a positive integer and set $h:=1 / m$. If $n=b m$ with $b$ an integer, then $u_{n}:=\Delta_{h}^{r} s_{n}$ is in $S_{n}$ and, with $g:=\Delta_{h}^{r} f$, we have

$$
\left\|g-u_{n}\right\|=o\left(n^{-k}\right) \text {. }
$$

Now any truncated power $t(x, y)=\left(x-x_{i}\right)_{+}{ }^{p} y^{q}$ or $t(x, y)=x^{p}\left(y-y_{j}\right)_{+}{ }^{q}$ in $T_{r, \rho}(\Sigma)$ is annihilated by $\Delta_{h}^{r}$. Hence, on $\mathbf{R}_{+}{ }^{2}, u_{n}$ is a linear combination of the splines $\Delta_{h}^{r} t$, with $t(x, y)=\left(x-x_{i}(n)\right)_{+}{ }^{p}\left(y-y_{j}(n)\right)_{+}{ }^{q}$ in $S_{n}$ and $i, j>0$. Such a $t$ has $\rho<p, q$ and $p+q<r$, and therefore $p, q<k$. Thus $u_{n}$ is a polynomial of coordinate order $k$ on each $\sigma_{i j}(n) \in \Sigma_{n}$ with $i, j \geqslant 0$.

For given $\eta>0$, choose $b$, so that $1 /((b+1) m) \leqslant k \eta<1 /(b m)$. Then the points $(i \eta, 0), 0 \leqslant i \leqslant k$, are in $\sigma_{00}(b m)$ and so $\left(\delta_{(\eta, 0)}^{k} u_{b m}\right)(0)=0$. Using $(2.1)$, we have

$$
\left|\left(\delta_{(\eta, 0)}^{k} g\right)(0)\right|=\left|\left(\delta_{(\eta, 0)}^{k}\left(g-u_{b m}\right)\right)(0)\right| \leqslant \text { const }\left\|g-u_{b m}\right\|_{\infty}=o\left((b m)^{-k}\right)=o\left(\eta^{k}\right),
$$


therefore $D^{(k, 0)} g(0)=0$. If we now let $m \rightarrow \infty$ and recall that $h=1 / m$ and $g=\Delta_{h}^{r} f$, we find $D^{(r+k, r)} f(0)=0$, as desired.

We now prove (ii). Without loss of generality we assume from now on that $f$ is supported in the unit cube $Q:=[0,1]^{2}$. If $\rho=\lfloor(r-3) / 2\rfloor$, then (ii) follows from the fact that $S_{n}$ contains the tensor product splines of order $k=r-\rho-1$. For general $\rho$, the argument is more involved. We need a certain subspace of $S_{n}$, the space $\tilde{S}_{r, \rho}\left(\Sigma_{n}\right)$ of those $s \in S_{n}$ for which $s_{\mid \sigma_{i j}(n)}$ agrees with a polynomial in the span of $\left\{x^{p} y^{q}: p+q<r, p, q<k\right\}$, all $i, j$. We will prove, by induction on $\rho$, the following

Claim. For any $\rho$ and any $r$ with $\rho \leqslant(r-3) / 2$ there is const so that for any $f \in C_{0}^{r}\left(\mathbf{R}^{2}\right)$ supported in $Q$ and any $n$, there is an $s \in \tilde{S}_{r, \rho}\left(\Sigma_{n}\right)$ supported in the square $(\rho+2)^{2} Q$ for which

$$
\|f-s\| \leqslant \text { const }\|f\|_{r} n^{-r+\rho+1} .
$$

This claim in turn gives (ii).

Certainly the claim is true when $\rho=-1$, the case of piecewise polynomial approximation (see, e.g. [5]). Suppose then that the claim has been established for all $\rho<\rho_{0}$ and consider $\rho=\rho_{0}>-1$. Take $r$ so that $\rho \leqslant(r-3) / 2$. Take $f \in C_{0}^{r}\left(\mathbf{R}^{2}\right)$ supported in $Q$, and let $g:=D^{(1,1)} f$. Then

$$
f(x, y)=\int_{0}^{x} \int_{0}^{y} g(\xi, \eta) d \xi d \eta \quad \text { for all }(x, y) \in \mathbf{R}_{+}{ }^{2},
$$

By induction hypothesis, we may choose $u \in \tilde{S}_{r-2, \rho-1}\left(\Sigma_{n}\right)$ so that the support of $u$ is in $(\rho+1)^{2} Q$ and

$$
\|g-u\| \leqslant \text { const }\|g\|_{r-2} n^{-(r-2)+(\rho-1)+1} \leqslant \text { const }\|f\|_{r} n^{-k+1} .
$$

Our approximation $s$ to $f$ is gotten by integrating $u$ and making local corrections using B-splines.

For $p=\left(p_{1}, p_{2}\right)$, let $\bar{M}_{p}(x, y):=M_{p_{1}}(x) M_{p_{2}}(y)$, with $M_{j}$ the univariate B-spline with knots $x_{j}(n), \ldots, x_{j+\rho+1}(n)$. Then $M_{j}$ is of order $\rho+1$ and smoothness $\rho-1$, and is supported on the interval $\left[x_{j}(n), x_{j+\rho+1}(n)\right]$. It follows that $\bar{M}_{p}$ is in $\tilde{S}_{r-2, \rho-1}\left(\Sigma_{n}\right)$. We assume $\bar{M}_{j}$ to be normalized to have integral 1 .

Consider the splines $N_{p}:=\bar{M}_{(\rho+1) p}$. Then $N_{p}$ is supported on the square

$$
Q_{p}:=\left[x_{(\rho+1) p_{1}}(n), x_{(\rho+1)\left(p_{1}+1\right)}(n)\right] \times\left[y_{(\rho+1) p_{2}}(n), y_{(\rho+1)\left(p_{2}+1\right)}(n)\right]
$$

and therefore the $N_{p}$ 's have disjoint support.

For each $p$, define $a_{p}:=\int_{Q_{p}}(g-u)$. From (2.4), it follows that

$$
\left|a_{p}\right| \leqslant\left|Q_{p}\right| \text { const }\|g\|_{r-2} n^{-k+1} \leqslant \text { const }\|f\|_{r} n^{-k-1} \text {. }
$$

Consider now the spline

$$
v:=u+\sum_{p} a_{p} N_{p}
$$

For each $p$, we have

$$
\int_{Q_{p}}(g-v)=0
$$


Hence, if we set

$$
s(x, y):=\int_{0}^{x} \int_{0}^{y} v(\xi, \eta) d \xi d \eta,
$$

then $s$ will be in $S_{r, \rho}\left(\Sigma_{n}\right)$ and if $(x, y) \in Q_{p}$ then

$$
f(x, y)-s(x, y)=\int_{0}^{x} \int_{0}^{y}(g-v)(\xi, \eta) d \xi d \eta=\int_{E}(g-v)
$$

with

$$
E:=\left([0, x] \times\left[y_{(\rho+1) p_{2}}(n), y\right]\right) \cup\left(\left[x_{(\rho+1) p_{1}}(n), x\right] \times[0, y]\right) .
$$

Using (2.6) and the fact that $f$ and $u$ have support in $\rho^{2} Q$, it follows that $s=0$ outside of $\left[(\rho+1)^{2}+(\rho+1)\right] Q \subseteq(\rho+2)^{2} Q$. Now, for any $(x, y) \in(\rho+2)^{2} Q$, we have $|E| \leqslant$ const $n^{-1}$. Further, $\int_{E} N_{p}$ is nonzero for at most $O(n)$ values of $p$. Therefore, (2.4) and (2.5) give

$$
\begin{aligned}
|f(x, y)-s(x, y)| & =\left|\int_{E}\left(g-u-\sum_{p} a_{p} N_{p}\right)\right| \\
& \leqslant\|g-u\||E|+\max _{p}\left|a_{p}\right| \sum_{p} \int_{E} N_{p} \\
& \leqslant \text { const }\|f\|_{r} n^{-k} .
\end{aligned}
$$

This proves (2.3) for the approximation just constructed.

3. Approximation on triangular grids. We now show how the results of the last section can be extended to triangular grids. We focus on the partition $\Delta$ which is gotten by dividing each square of $\Sigma$ into two triangles by adding the northeast diagonal. It will be clear, however, that our techniques apply to more general partitions, a point made in $\$ 4$. We begin by developing methods for constructing smooth spline approximants. This turns out to be the more significant part of the problem. Our construction will be based on some variants of the ideas of multivariate B-splines.

Let $p<m$ and let $P$ denote the projection of $\mathbf{R}^{m}$ onto $\mathbf{R}^{p}$, i.e., $z=(P z, y)$ for $z \in \mathbf{R}^{m}$. If $A$ is a simplex in $\mathbf{R}^{m}$ with vertices $v_{0}, \ldots, v_{m}$, then the function

$$
M(x):=\operatorname{vol}_{m-p}\{z \in A: P z=x\}, \quad x \in \mathbf{R}^{p},
$$

is a piecewise polynomial $[4,8]$ of order $m-p+1$ which is, up to a constant (viz., $\operatorname{vol}_{m}(A)$ ), completely determined by the points $P v_{0}, \ldots, P v_{m}$ in $\mathbf{R}^{p}$ and has its support in their convex hull.

When $p=2$, the piecewise polynomial $M$ has any segment connecting any two of the projected vertices $P v_{0}, \ldots, P v_{m}$ as a mesh line, and is a polynomial on any connected set not intersected by such a mesh line. For many purposes (including ours), this results in too complicated a grid in the plane. This can be avoided if we replace the simplex $A$ in (3.1) by an appropriate $m$-dimensional convex polyhedron $B$ and so define

$$
M_{B}(x):=\operatorname{vol}_{m-p}\{z \in B: P z=x\}, \quad x \in \mathbf{R}^{p} .
$$


The function $M_{B}$ so defined is again piecewise polynomial of total order $m-p+1$ because $B$ can be decomposed into disjoint simplices and therefore $M_{B}$ is a sum of (simplicial) B-splines. It has its support in $P(B)$.

A more useful analytic description of $M_{B}$ is given by the identity (used first in the context of simplicial B-splines by Micchelli, see [8])

$$
\int_{\mathbf{R}^{p}} \phi(x) M_{B}(x) d x=\int_{B} \phi(P z) d z
$$

which holds for any test function $\left(\phi \in C_{0}^{\infty}\right)$. The right-hand side defines $M_{B}$ as a distribution on $\mathbf{R}^{p}$. One of the advantages of (3.3) is that it can be used to define $M_{B}$ when $B$ is a convex polyhedron of dimension $q<m$. The right-hand side is then interpreted as a $q$-dimensional surface integral. This definition also makes sense when $P(B)$ has dimension $<p$. In this case, $M_{B}$ is defined only as a distribution.

Suppose then that $B$ is a convex polyhedron of dimension $q \leqslant m$. As is pointed out in [2], it is easy to check the smoothness of $M_{B}$ by using the differentiation formula

$$
D_{P z} M_{B}=-\Sigma\left(z \cdot n_{i}\right) M_{B_{i}}
$$

proved there. Here, the $B_{i}$ are the facets of $B$, i.e., the faces of dimension $q-1$, which make up the boundary of $B$, and $n_{i}$ are their respective normals in the affine hull of $B$. Repeated application of (3.4) shows that $D^{(\alpha, \beta)} M_{B}$ can be written as a linear combination of $M_{F}$ 's where each $F$ is a face of $B$ of dimension $q-\alpha-\beta$. In particular, if $\operatorname{dim} P(F)=p$ for all faces, then $D^{(\alpha, \beta)} M_{B}$ is again a piecewise polynomial. Thus $M_{B}$ is in $C^{q-d-2}$, with $d$ the largest integer for which there is a face $F$ of $B$ of dimension $d$ with $P(F)$ of dimension $<p$. Note also that the discontinuities of $M_{B}$ occur across the $(p-1)$-dimensional sets $P(F)$, with $F$ a face of $B$.

We now turn to the construction of spline approximants on the triangular partition $\Delta$ of $\mathbf{R}^{2}$. Let $e_{i}:=\left(\delta_{i j}\right)_{j=1}^{m}$ be the unit coordinate vectors in $\mathbf{R}^{m}$ and define

$$
\begin{aligned}
& v_{1}:=e_{1}, \quad v_{2}:=e_{2}, \\
& v_{j}:=e_{j}\left\{\begin{array}{ll}
e_{1}, & \text { if } j \equiv 1(\bmod 3) \\
e_{2}, & \text { if } j \equiv 2(\bmod 3) \\
e_{1}+e_{2}, & \text { if } j \equiv 3(\bmod 3)
\end{array}\right\}, \quad j=3,4, \ldots, m \text {. }
\end{aligned}
$$

The vectors $v_{1}, \ldots, v_{m}$ determine a parallelepiped $B$ with vertices $\sum_{1}^{m} \varepsilon_{i} v_{i}$, where $\varepsilon_{i} \in\{0,1\}$, all $i$. The translated parallelepipeds $B_{i j}:=i e_{1}+j e_{2}+B$ have pairwise disjoint interiors and fill out the slab $\mathbf{R}^{2} \times[0,1]^{m-2}$, hence form a partition for that slab.

Consider the functions $M:=M_{B}$ and $M_{i j}(x, y):=M(x-i, y-j)=$ $M_{B_{i j}}(x, y)$ defined by (3.3) (or, equivalently, by (3.2)) with $p=2$.

LEMMA 2. (i) $\Sigma M_{i j}=1$ on $\mathbf{R}^{2}$.

(ii) $M_{i j} \in S_{r, \rho}(\Delta)$ for $r:=m-1$ and $\rho:=\lfloor(2 r-4) / 3\rfloor$. 
Proof. The identity (i) follows from the representation (3.2) and the fact that $\cup B_{i j}=\mathbf{R}^{2} \times[0,1]^{m-2}$, with the $B_{i j}$ having pairwise disjoint interiors.

We have already noted that $M$ is a piecewise polynomial of total order $m-1=r$. To check the smoothness of $M$, note that any face $F$ of $B$ is of the form

$$
F=\left\{v+\sum_{i \in I} \alpha_{i} v_{i}: 0 \leqslant \alpha_{i} \leqslant 1\right\}
$$

with $v$ some vertex of $B$ and $I \subseteq \mathbf{m}$. If $P(F)$ is a segment, i.e., $P(F)$ has dimension 1 , then all $i \in I$ are congruent modulo 3 , hence $|I| \leqslant\lceil m / 3]$. Therefore, $M$ has smoothness $\rho:=m-\lceil m / 3\rceil-2=\lfloor(2 r-4) / 3\rfloor$. Such a segment $P(F)$ must lie on the line

$$
P(v)+x P\left(v_{j}\right), \quad-\infty<x<\infty,
$$

with $j \equiv i(\bmod 3)$, all $i \in I$, and $j \in 3$. This is one of the mesh lines which form the partition $\Delta$. Since $M$ is discontinuous only across such segments $P(F)$, it follows that $M$ is in $S_{r, \rho}(\Delta)$, and therefore so are all the $M_{i j}$.

LEMMA 3. If $\rho>(2 r-4) / 3$, then every $s \in S_{r, \rho}(\Delta)$ can be represented on $\mathbf{R}_{+}{ }^{2}$ as a linear combination of the truncated powers in

$$
\begin{aligned}
T_{r, \rho}(\Delta):=\left\{x^{p} y^{q},\left(x-x_{i}\right)_{+}{ }^{p} y^{q}, x^{p}\left(y-y_{j}\right)_{+}^{q},\left(x-y-x_{i}\right)_{+}{ }^{p}(x+y)^{q}:\right. \\
p, q \geqslant 0 ; p+q<r ; \\
p, q>\rho \text { whenever they exponentiate a truncated function }\} .
\end{aligned}
$$

Proof. Denote by $\sigma_{i j}^{-}$and $\sigma_{i j}^{+}$the left, respectively right, triangle in $\Delta$ which make up the square $\sigma_{i j}$. Let $s \in S$ and let $t_{0}$ be the polynomial of order $r$ which agrees with $s$ on $\sigma_{00}^{-}$. Then $s-t_{0}=0$ on the line $y=x$. Therefore $s-t_{0}=u$ on $\sigma_{00}^{+}$, with

$$
u(x, y)=\sum_{\substack{p>\rho \\ p+q<r}} c_{p q}(x-y)^{p}(x+y)^{q} .
$$

Set $t_{1}:=t_{0}+\Sigma c_{p q}(x-y)_{+}{ }^{p}(x+y)^{q}$. Then $s=t_{1}$ on $\sigma_{00}$. We now continue in this manner, moving to the right, using the truncated powers $\left(x-x_{i}\right)_{+}{ }^{p} y^{q}$ and $\left(x-y-x_{i}\right)_{+}{ }^{p}(x+y)^{q}$ to construct a function $t$ in the span of $T_{r, \rho}(\Delta)$ which agrees with $s$ on $\sigma_{i 0}, i=0,1,2, \ldots$ On the first column $\left(\sigma_{0 i}\right)_{i=1}^{\infty}$ of squares, this $t$ is just the polynomial $t_{0}$. Proceeding now up that column, we further modify $t$ by the addition of a suitable linear combination of the truncated powers $x^{p}\left(y-y_{i}\right)_{+}{ }^{q}$ and $(x+y)^{p}\left(y-x-y_{j}\right)_{+}{ }^{q}$ (note that $\left(y-x-y_{j}\right)_{+}{ }^{q}=\left(y-x-x_{i}\right)^{q}\left(x-y+x_{j}\right)_{+}{ }^{q}$ for suitable $x_{i}$ ) and so obtain a new $t$ in the span of $T$ which agrees with $s$ on the first row and first column of squares, i.e., on

$$
\bigcup_{i=0}^{\infty} \sigma_{i 0} \cup \sigma_{0 i} \text {. }
$$

We claim that now $s=t$ on all of $\mathbf{R}_{+}{ }^{2}$. We prove this by showing that $s=t$ on $\sigma_{i j}$, $i, j>0$, using lexicographic ordering and induction. 


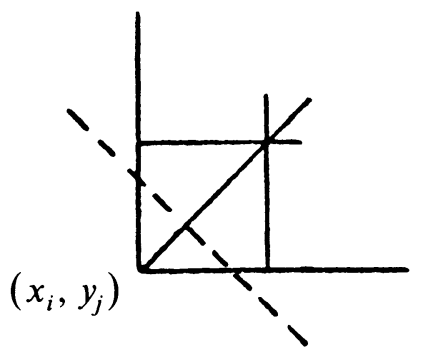

Assume that $s=t$ on $\sigma_{\mu \nu}$ for all $\mu<i$ and for all $(\mu, \nu)$ with $\mu=i$ and $\nu<j$ (as is the case for $(i, j)=(1,1))$. Then $u:=s-t$ is a piecewise polynomial function of total order $r$ and smoothness $\rho$ which vanishes for $0<x<x_{i}$ and for $0<y<y_{j}$. If now $u$ were nonzero somewhere in $\sigma_{i j}$, then, on restricting $u$ to some line $x+y=c$, with $x_{i}+y_{j}<c<x_{i+1}+y_{j}$, we would obtain a univariate piecewise polynomial function $v$ of order $r$ and smoothness $\rho$ which vanishes outside some interval $[a, b]$ and has just three knot locations, viz. the points $a,(a+b) / 2$, and $b$. Further, $v$ would be nonzero somewhere in $[a, b]$. This would imply that the sum of the multiplicities of the knots $a,(a+b) / 2$, and $b$ is at least $r+1$, which would imply that at least one knot has multiplicity $\geqslant(r+1) / 3$, therefore $\rho \leqslant r-1$ $-\lceil(r+1) / 3\rceil=\lfloor(2 r-4) / 3\rfloor$, a contradiction. This advances the induction hypothesis and so finishes the proof.

TheOREM 3. $\cup_{n} S_{r, \rho}\left(\Delta_{n}\right)$ is dense in $C_{0}\left(\mathbf{R}^{2}\right)$ if and only if $\rho \leqslant(2 r-4) / 3$.

Proof. By Lemma 2, $S_{r, \rho}(\Delta)$ contains the local and positive partition of unity $\left(M_{i j}\right)$ as long as $\rho \leqslant(2 r-4) / 3$. This implies the density of $\cup_{n} S_{r, \rho}\left(\Delta_{n}\right)$ by the following standard argument: The simple approximation map

$$
T f:=\sum_{i, j} f\left(x_{i}, y_{j}\right) M_{i j}
$$

carries $f$ into $S_{r, \rho}(\Delta)$ and satisfies

$$
\begin{aligned}
|(f-T F)(x, y)| & =\left|\sum_{i, j}\left(f(x, y)-f\left(x_{i}, y_{j}\right)\right) M_{i j}(x, y)\right| \\
& \leqslant \max _{M_{i j}(x, y) \neq 0}\left|f(x, y)-f\left(x_{i}, y_{j}\right)\right| .
\end{aligned}
$$

Consequently, $\|f-T f\| \leqslant \omega(f ; \operatorname{diam} \operatorname{supp} M)$. Now scale.

For the converse, assume that $\rho>(2 r-4) / 3$. Consider the difference operator $\Lambda_{h}:=\Delta_{h}^{r} \cdot \delta_{(h, h)}^{r}$. Since $\Delta_{h}^{r}$ and $\delta_{(h, h)}^{r}$ commute, we have $\Lambda_{h} t=0$ for all $t$ in the span of $T_{r, \rho}(\Delta)$ defined in Lemma 3. Therefore, by Lemma $3, \Lambda_{h} s=0$ on $\mathbf{R}_{+}^{2}$ for every $s \in S_{r, \rho}(\Delta)$.

Suppose now that $f \in C_{0}^{\infty}\left(\mathbf{R}^{2}\right)$ and that $\left\|f-s_{n}\right\| \rightarrow 0$ (as $n \rightarrow \infty$ ) for some $s_{n} \in S_{r, \rho}\left(\Delta_{n}\right), n=1,2, \ldots$. Assume without loss of generality that $f$ has its support in $\mathbf{R}_{+}{ }^{2}$. Since $\Delta_{n}$ is obtained from $\Delta$ by scaling, it follows that $\Lambda_{h} s_{n}=0$ on $\mathbf{R}_{+}{ }^{2}$, 
therefore $\Lambda_{h} f=0$. Dividing by $h^{3 r}$ and taking the limit as $h \rightarrow 0$ shows that

$$
\left(D_{e_{1}} D_{e_{2}} D_{e_{1}+e_{2}}\right)^{r} f=0 \text {, }
$$

which shows that $f$ is not an arbitrary function in $C_{0}^{\infty}\left(\mathbf{R}^{2}\right)$.

Next we consider the approximation from $S_{r, \rho}\left(\Delta_{n}\right)$ to smooth functions. For this, we need to consider the B-splines associated with faces of $B$. Any $I \subseteq \mathbf{m}$ is associated with a face, viz. the face

$$
F:=F_{I}:=\left\{\sum_{i \in I} \alpha_{i} v_{i}: 0 \leqslant \alpha_{i} \leqslant 1\right\} .
$$

This is a face of dimension $|I|$, or a $|I|$-face, for short.

Denote the corresponding B-spline $M_{F_{I}}$ by $M^{I}$. We are particularly interested in faces for which

$$
2 \subseteq I \text { and }|I|>2
$$

For such a face, set

$$
Q:=\left\{\sum_{i \notin I} \alpha_{i} e_{i}: 0 \leqslant \alpha_{i} \leqslant 1\right\} .
$$

Then, for any test function $\phi$,

$$
\int_{\mathbf{R}^{2}} \phi M^{I}=\int_{F} \phi \circ P=\int_{F+Q} \phi \circ P,
$$

using the facts that $\phi(P z)=\phi\left(P z_{0}\right)$ in case $z \in z_{0}+Q$, and that the $(m-|I|)$ dimensional volume of $Q$ is 1 . This shows that, for such a face,

$$
M^{I}(x, y)=\operatorname{vol}_{m-2}\{z \in F+Q: P z=(x, y)\}=\operatorname{vol}_{|I|-2}\left\{z_{0} \in F: P z_{0}=(x, y)\right\} .
$$

Now define

$$
M_{i j}^{I}:=M_{F_{i j}},
$$

with $F_{i j}:=(i, j)+F$ the face $F$ translated. The $F_{i j}$ have pairwise disjoint interiors and form the set $\mathbf{R}^{2} \times C$ with $C:=\left\{\sum_{i \in I \backslash 2} \alpha_{i} v_{i}: 0 \leqslant \alpha_{i} \leqslant 1\right\}$. Hence it follows from (3.5) that

$$
\sum_{i, j} M_{i j}^{I}=\operatorname{vol}_{|I|-2}(C)=1
$$

Our next lemma is a special case of a result in [3].

LEMMA 4. Let $r:=m-1$ and $k:=\lfloor(2 r+2) / 3\rfloor$. Then, for $\alpha+\beta<k$, there are polynomials $Q_{\alpha \beta}$ of the form $Q_{\alpha \beta}(x, y)=x^{\alpha} y^{\beta} /(\alpha ! \beta !)+R_{\alpha \beta}(x, y)$ with $R_{\alpha \beta}$ of coordinate order $(\alpha, \beta)$ such that

$$
\sum_{i, j} Q_{\alpha \beta}(i, j) M_{i j}(x, y)=\frac{x^{\alpha} y^{\beta}}{\alpha ! \beta !} .
$$


Proof. The proof is an adaptation of the argument in an early draft of [3] to our particular context. It is essentially an inductive proof on the number $|I|$ of the following

Claim. Let $\mathbf{2} \subseteq I \subseteq \mathbf{m}$. Let $h:=h_{I}:=|I|-d$, with $d$ the largest integer for which there is a d-dimensional face $G$ of $F=F_{I}$ with $\operatorname{dim} P(G)<2$. Then, for any $\alpha+\beta<h$, the function $\phi_{\alpha \beta}$, given by

$$
\phi_{\alpha \beta}:=\sum_{i, j} i^{\alpha} j^{\beta} M_{i j}^{I},
$$

is a polynomial of coordinate degree $(\alpha, \beta)$ with leading coefficient 1 , i.e.,

$$
\phi_{\alpha \beta}(x, y)=x^{\alpha} y^{\beta}+\text { terms of order }(\alpha, \beta) .
$$

To prove this claim, consider first the case $|I|=3$. Let $I=\{1,2, \rho\}$. If $\rho \equiv 1$ or 2 $(\bmod 3)$, then $d=2$, hence $h=1$ and the claim is just (3.6). If $\rho \equiv 3(\bmod 3)$, then $M^{I}$ vanishes at all mesh points $(i, j)$ except that $M^{I}(1,1)=1$, while $\Sigma_{i j} \alpha_{i j} M_{i j}^{I}$ is continuous and piecewise linear. Therefore

$$
\sum_{i, j} f(i+1, j+1) M_{i j}^{I}=f
$$

for all linear functions $f$, and the claim follows also for this case.

Let now $s>3$, assume the claim proved for all $|I|<s$, and consider some $I$ with $|I|=s$.

If $d=|I|-1$, then $h=1$ and the claim reduces once again to (3.6). Otherwise, $d<|I|-1$. In this case, consider $\mu \in I \backslash 2$ and set $u:=v_{\mu}$. We calculate $D_{P u} \phi_{\alpha \beta}$ for any $\alpha+\beta<h$. By (3.4),

$$
D_{P u} M^{I}=-\sum\left(u \cdot n_{i}\right) M_{F_{i}}
$$

where the $F_{i}$ are the $(|I|-1)$-faces of $F_{I}$ and the $n_{i}$ are the corresponding outward normals (in the affine hull of $\left.F_{I}\right)$. This implies that $\left(u \cdot n_{i}\right)=0$ for any face $F_{i}$ parallel to $u$. There are only two faces which are not parallel to $u$, viz. the faces $F_{J}$ and $F_{J}+u$, with

$$
J:=I \backslash\{u\} .
$$

Since their normals sum to zero and $\left|\left(u \cdot n_{i}\right)\right|=1,(3.9)$ becomes simply

$$
D_{P u} M^{I}=M^{J}-M_{P u}^{J} \text {. }
$$

This implies that

$$
D_{P u} \phi_{\alpha \beta}=\sum_{i, j} f_{\alpha \beta}(i, j) M_{i j}^{J}
$$

with $f_{\alpha \beta}(x, y):=x^{\alpha} y^{\beta}-\xi^{\alpha} \eta^{\beta}$ and $(\xi, \eta):=(x, y)-P u$. More explicitly,

$$
\text { if } P u=\left\{\begin{array}{c}
e_{1} \\
e_{2} \\
e_{1}+e_{2}
\end{array}\right\} \quad \text { then } f_{\alpha \beta}(i, j)=\left\{\begin{array}{l}
\alpha i^{\alpha-1} j^{\beta} \\
\beta i^{\alpha} j^{\beta-1} \\
\alpha i^{\alpha-1} j^{\beta}+\beta i^{\alpha} j^{\beta-1}
\end{array}\right\}
$$

+ lower coord. order terms. 
Since $h_{J} \geqslant h-1$ and $|J|<|I|$ and $f_{\alpha \beta}$ is of coordinate degree $<(\alpha, \beta)$, we may apply the induction hypothesis to conclude that $D_{P u} \phi_{\alpha \beta}$ is a polynomial; more precisely, that

$D_{P u} \phi_{\alpha \beta}(x, y)=\left\{\begin{array}{l}\alpha x^{\alpha-1} y^{\beta} \\ \beta x^{\alpha} y^{\beta-1} \\ \alpha x^{\alpha-1} y^{\beta}+\beta x^{\alpha} y^{\beta-1}\end{array}\right\}+$ lower order terms, if $P u=\left\{\begin{array}{c}e_{1} \\ e_{2} \\ e_{1}+e_{2}\end{array}\right\}$.

Integrating back up, we find that

$$
\phi_{\alpha \beta}(x, y)=x^{\alpha} y^{\beta}+\text { lower order terms }+g_{\alpha \beta}\left(z_{1} x+z_{2} y\right)
$$

with $g_{\alpha \beta}$ a univariate function and $z \in \mathbf{R}^{2}$ perpendicular to $P u$.

But now, since $|I|>3$ and $d<|I|-1$, it is possible to choose $u$ in different ways, say $u=v$ and $u=w$, so that $P v$ and $P w$ are linearly independent. This allows the conclusion that actually (3.8) holds and so advances the induction hypothesis.

This finishes the proof of the claim. Taking now, in particular, $I=\mathbf{m}$, we find that $d=\lceil m / 3\rceil$, hence $h_{I}=m-\lceil m / 3\rceil=\lfloor 2 m / 3\rfloor=k$, and we conclude that (3.8) holds for all $\alpha+\beta<k$. Repeated application of this fact gives us, for each $\alpha+\beta<k$, a polynomial $Q_{\alpha \beta}$ of coordinate degree $(\alpha, \beta)$ with leading coefficient $1 /(\alpha ! \beta !)$ such that

$$
\sum_{i, j} Q_{\alpha \beta}(i, j) M_{i j}(x, y)=\frac{x^{\alpha} y^{\beta}}{\alpha ! \beta !} .
$$

We conclude that, for any $r, s$, and any $\alpha+\beta<k$,

$$
\begin{aligned}
\frac{(x-r)^{\alpha}(y-s)^{\beta}}{\alpha ! \beta !} & =\sum_{i, j} Q_{\alpha \beta}(i-r, j-s) M_{i-r, j-s}(x-r, y-s) \\
& =\sum_{i, j} Q_{\alpha \beta}(i-r, j-s) M_{i j}(x, y) .
\end{aligned}
$$

Consequently, for any $p \in \mathbf{P}_{k}$,

$$
\begin{aligned}
p(x, y) & =\sum_{\alpha+\beta<k} \frac{(x-r)^{\alpha}(y-s)^{\beta}}{\alpha ! \beta !}\left(D^{(\alpha, \beta)} p\right)(r, s) \\
& =\sum_{i, j} q_{r s}(i, j) M_{i j}(x, y)
\end{aligned}
$$

with

$$
q_{r s}(x, y):=\sum_{\alpha+\beta<k} Q_{\alpha \beta}(x-r, y-s)\left(D^{(\alpha, \beta)} p\right)(r, s) .
$$

The next lemma implies that $q_{r s}$ is, in fact, independent of $r$ and $s$.

LeMma 5. The map $\mathbf{P}_{k} \rightarrow \operatorname{span}\left(M_{i j}\right): q \mapsto \Sigma_{i, j} q(i, j) M_{i j}$ is one-one.

Proof. Suppose $q \in \mathbf{P}_{k} \backslash\{0\}$. Then, for any $r>0$, there exists $c \in \mathbf{R}^{2}$ so that $q$ is of one strict sign on the ball $B_{r}(c)$ of radius $r$ and center $c$. Choose $r>\operatorname{diam} \operatorname{supp}(M)$. Since

$$
(i, j) \in \operatorname{supp}\left(M_{i j}\right)^{-}=(i, j)+\operatorname{supp}(M)^{-},
$$


it follows that $\left\{q(i, j) M_{i j}(c): M_{i j}(c) \neq 0\right\}$ are all of one strict sign, hence

$$
\sum_{i, j} q(i, j) M_{i j} \neq 0
$$

We conclude that $q_{r s}$ in (3.11) does not depend on $r, s$. In particular,

$$
q_{r s}(i, j)=q_{i j}(i, j)=\sum_{\alpha+\beta<k} Q_{\alpha \beta}(0,0)\left(D^{(\alpha, \beta)} p\right)(i, j) .
$$

Corollary. For any $p \in \mathbf{P}_{k}, p=\Sigma_{i, j} \lambda(p(\cdot+i, \cdot+j)) M_{i j}$ with

$$
\lambda f:=\sum_{\alpha+\beta<k} Q_{\alpha \beta}(0,0)\left(D^{(\alpha, \beta)} f\right)(0,0) .
$$

We use the linear functional $\lambda$ to define an approximation from $S_{r, \rho}(\Delta)$ in the now standard quasi-interpolant fashion. First, we modify $\lambda$ so as to make it applicable to any $f \in C_{0}\left(\mathbf{R}^{2}\right)$. For this, let $\mu$ be a bounded extension of $\lambda$ from $\mathbf{P}_{k}$ to $C\left(\sigma_{00}^{+}\right)$. Then $\mu$ can be taken to be a bounded linear functional on all of $C_{0}\left(\mathbf{R}^{2}\right)$. In this way, we obtain a linear map

$$
L f:=\sum_{i, j} \mu(f(\cdot+i, \cdot+j)) M_{i j}
$$

on $C_{0}\left(\mathbf{R}^{2}\right)$ to $S_{r, \rho}(\Delta)$ which is local, reproduces $\mathbf{P}_{k}$, and is bounded by $\|\mu\|$ since $\sum_{i j} M_{i j}=1$. This implies that

$$
\|f-L f\|(A) \leqslant(\|\mu\|+1) \operatorname{dist}_{N(A)}\left(f, \mathbf{P}_{k}\right)
$$

with $N(A):=\bigcup\left\{\operatorname{supp}\left(M_{i j}\right): \operatorname{supp}\left(M_{i j}\right) \cap A \neq \varnothing\right\}$. Scaling by $1 / n$ to get to the partition $\Delta_{n}$ gives the map $L_{n}$ to $S_{r, \rho}\left(\Delta_{n}\right)$ for which we have the following

THEOREM 4. Set $m:=r-1, \rho:=\lfloor(2 r-4) / 3\rfloor$ and $k:=\lfloor(2 r+2) / 3\rfloor=\rho+$ 2. If $f \in C_{0}\left(\mathbf{R}^{2}\right)$, then $L_{n} f \in S_{r, \rho}\left(\Delta_{n}\right)$ and

$$
\left\|f-L_{n} f\right\| \leqslant \text { const }_{k} \omega_{k}(f, 1 / n), \quad n=1,2, \ldots,
$$

with $\omega_{k}$ the kth order modulus of smoothness of $f$. In particular, if $f \in C_{0}^{k}\left(\mathbf{R}^{2}\right)$, then

$$
\left\|f-L_{n} f\right\| \leqslant \text { const }_{k}|f|_{k} n^{-k} .
$$

Proof. Let $A=\sigma_{i j}(n)$ and, correspondingly

$$
N(A):=\bigcup\left\{\operatorname{supp}\left(M_{p q n}\right): \operatorname{supp}\left(M_{p q n}\right) \cap A \neq \varnothing\right\} .
$$

By (3.12),

$$
\left\|f-L_{n} f\right\|(A) \leqslant\left(\text { const }_{r}+1\right) \operatorname{dist}_{N(A)}\left(f, \mathbf{P}_{k}\right),
$$

while, e.g., from [5],

$$
\operatorname{dist}_{N(A)}\left(f, \mathbf{P}_{k}\right) \leqslant \text { const }_{k} \varepsilon
$$

with $\varepsilon=\omega_{k}(f, 1 / n)$ or $\varepsilon=|f|_{k} n^{-k}$ since diam $N(A)=O(1 / n)$.

4. Concluding remarks. The construction of smooth multivariate spline interpolants developed in $\$ 3$ can be extended to more general partitions (see also [3]). Suppose that $\Pi$ is a partition of $\mathbf{R}^{2}$ which can be obtained as follows. Starting with a regular partition $\Pi_{0}$ associated with the two independent directions $d_{1}$ and $d_{2}$, we 
add mesh lines through all the vertices of $\Pi_{0}$ in the directions of the vectors $d_{3}, \ldots, d_{p}$, with these $d_{i}$ 's nonzero vertices of $\Pi_{0}$ other than $d_{1}$ or $d_{2}$, and pairwise linearly independent.

Thus $\Sigma$ is associated with the directions $e_{1}$ and $e_{2}, \Delta$ is associated with the directions $e_{1}, e_{2}$ and $e_{1}+e_{2}$, while the four directions $e_{1}, e_{2}, e_{1}+e_{2}, e_{1}-e_{2}$ are associated with a partition in which both diagonals are drawn into every square.

Given such a partition $\Pi$ and $m \geqslant p$, define vectors $v_{1}, \ldots, v_{m}$ in $\mathbf{R}^{m}$ by $v_{1}:=d_{1}, v_{2}:=d_{2}$, and, for $i>2, v_{i}:=d_{j}+e_{i}$, with $j \in \mathbf{p}$ and $j=i(\bmod p)$. Set $B:=\left\{\sum_{1}^{m} \alpha_{i} v_{i}: 0 \leqslant \alpha_{i} \leqslant 1\right\}$. Then the corresponding B-spline $M_{B}$ given by (3.3) is of order $r:=m-1$ and smoothness $\rho:=r-1-\lceil(r+1) / p\rceil$. If $\left(x_{i}, y_{i}\right)$ are the vertices of $\Pi_{0}$, then the translated splines $M_{i}$ given by

$$
M_{i}(x, y):=M_{B}\left(x-x_{i}, y-y_{i}\right),
$$

all $i$, form a local partition of unity. Using arguments like those for Lemma 4 and Theorem 4, they can be used to construct a local and bounded quasi-interpolant on $C_{0}\left(\mathbf{R}^{2}\right)$ into $S_{r, \rho}\left(\Pi_{n}\right)$ which reproduces polynomials of total order $k:=\rho+2$, hence approximates $C_{0}^{k}\left(\mathbf{R}^{2}\right)$-functions to within $O\left(n^{-\mathbf{k}}\right)$.

The particular choice $d_{j}:=(\cos \pi j / 3, \sin \pi j / 3), j=1,2,3$, results in a partition of $\mathbf{R}^{2}$ into equilateral triangles. We have recently learned that P. Frederickson [6, 7] has studied spline approximation on this partition and has shown the existence of spline interpolants from $S_{r, \rho}\left(\Pi_{n}\right)$ in the case $\rho=(2 r-4) / 3$ and $r \equiv 2(\bmod 3)$. This is accomplished by using a partition of unity given as translates of a fixed finite support spline obtained by a certain convolution.

\section{REFERENCES}

1. C. de Boor and G. Fix, Spline approximation by quasi-interpolants, J. Approx. Theory 7 (1973), $19-45$.

2. C. de Boor and K. Höllig, Recurrence relations for multivariate B-splines (MRC TSR \#2215, 1981), Proc. Amer. Math. Soc. 85 (1982), 397-400.

3. _ B-splines from parallelepipeds, MRC TSR \#2320, 1982.

4. W. Dahmen, On multivariate B-splines, SIAM J. Numer. Anal. 17 (1980), 179-191.

5. W. Dahmen, R. DeVore and K. Scherer, Multi-dimensional spline approximation, SIAM J. Numer. Anal. 17 (1980), 380-402.

6. P. O. Frederickson, Generalized triangular splines, Math. Report 7-71, Lakehead University, 1971.

7. Q Quasi-interpolation, extrapolation, and approximation on the plane, Proc. Manitoba Conf. on Numerical Mathematics (Winnipeg, 1971), pp. 159-176.

8. C. A. Micchelli, On a numerically efficient method for computing multivariate $B$-splines, Multivariate Approximation Theory (W. Schempp and K. Zeller, eds.), Birkhäuser, Basel, 1979, pp. 211-243.

9. P. Sablonniere, De l'existence de spline à support borné sur une triangulation équilatérale du plan, Publication ANO-39, U.E.R. d'I.E.E.A.-Informatique, Université de Lille I, February 1981.

Mathematics Research Center, University of Wisconsin, MAdison, Wisconsin 53705

Department of Mathematics and Statistics, University of South Carolina, Columbia, South Carolina 29208 